\title{
TAUT AND TIGHT COMPLEX MANIFOLDS
}

\section{THEODORE J. BARTH}

Abstract. Taut and tight manifolds, introduced recently by $\mathrm{H}$. $\mathrm{Wu}$, are characterized as follows. Let $D$ denote the open unit disk in $C$. The complex manifold $N$ is taut iff the set $A(D, N)$ of holomorphic maps from $D$ into $N$ is a normal family. If $d$ is a metric inducing the topology on $N,(N, d)$ is tight iff $A(D, N)$ is equicontinuous. It is also shown that every taut manifold is tight in a suitable metric.

By convention, all complex manifolds are connected and second countable. For complex manifolds $M$ and $N, A(M, N)$ denotes the set of holomorphic maps from $M$ into $N$. According to Wu [3, Definitions 1.2 and 1.3, p. 199], $N$ is called taut iff $A(M, N)$ is normal for all $M$. If $d$ is a metric inducing the topology on $N,(N, d)$ is called tight iff $A(M, N)$ is equicontinuous for all $M$.

Let \|\| denote the euclidean norm in $C^{m}$ and $D^{m}=\{z \mid\|z\|<1\} \subset C^{m}$ be the unit ball.

THEOREM 1. Let $d$ be a metric inducing the topology on the complex manifold $N$. If $A(D, N)$ is equicontinuous, then $(N, d)$ is tight.

Proof. Suppose that $(N, d)$ is not tight. Then there exists a complex manifold $M$ such that $A(M, N)$ is not equicontinuous. This means that there exist $\epsilon_{0}>0, m_{0} \in M$, and sequences $\left\{m_{k}\right\}$ in $M,\left\{f_{k}\right\}$ in $A(M, N)$ such that $m_{k} \rightarrow m_{0}$ as $k \rightarrow \infty$ and $d\left(f_{k}\left(m_{k}\right), f_{k}\left(m_{0}\right)\right) \geqq \epsilon_{0}$ for all $k$. By going to local coordinates, we may assume that $m_{0}=0 \in D^{m}$ $\subset M$ and that $m_{k} \in D^{m}$ for all $k$. For $z \in D$, define $g_{k}(z)=f_{k}\left(z m_{k} /\left\|m_{k}\right\|\right)$. Then $g_{k} \in A(D, N),\left\|m_{k}\right\| \rightarrow 0$ as $k \rightarrow \infty$, and $d\left(g_{k}\left(\left\|m_{k}\right\|\right), g_{k}(0)\right)$ $=d\left(f_{k}\left(m_{k}\right), f_{k}\left(m_{0}\right)\right) \geqq \epsilon_{0}$ for all $k$. Thus $A(D, N)$ is not equicontinuous.

For topological spaces $M$ and $N$, let $C(M, N)$ denote the space of continuous maps from $M$ into $N$ endowed with the compact-open topology $\left[1\right.$, p. 221]. Let $N^{*}=N \cup\{\infty\}$ be the one point compactification of $N[1$, p. 150]. Abusing notation, $\infty$ also denotes the maps into $N^{*}$ which are identically $\infty$.

Lemma 1. Let $N$ be a connected locally compact metric space. Then $N$ is second countable and $N^{*}$ is metrizable. 1969.

Presented to the Society, January 24, 1970; received by the editors August 12,

AMS Subject Classifications. Primary 3240, 3260, 5760; Secondary 3030.

Key Words and Phrases. Taut manifold, tight manifold, normal family, equicontinuous family. 
Proof. According to [2, Lemma 3, pp. 269-271], $N$ is second countable. Since $N$ is locally compact, $N^{*}$ is second countable. By the Urysohn metrization theorem [1, Theorem 16, p. 125], $N^{*}$ is metrizable.

Lemma 2. Let $M$ and $N$ be connected locally compact metric spaces, and let $F$ be a closed subset of $C(M, N)$. Then $F$ is a normal family if and only if $F \cup\{\infty\}$ is a compact subset of $C\left(M, N^{*}\right)$.

Proof. Since $M$ and $N^{*}$ are second countable (Lemma 1 ), so is $C\left(M, N^{*}\right)$. Thus compact means sequentially compact. The conclusion then follows from the definition of a normal family [3, Definition 1.1, p. 197].

Lemma 3. Let $M$ be a locally compact metric space, and let $N_{0}$ be a compact metric space. Then a subset of $C\left(M, N_{0}\right)$ is compact if and only if it is closed and equicontinuous.

Proof. This is a special case of the Ascoli theorem [1, Theorem 17, pp. 233-234].

Theorem 2. Let $N$ be a complex manifold. If $A(D, N)$ is a normal family, then $N$ is taut.

Proof. By Lemma $1, N^{*}$ is metrizable. Let $d$ be a metric inducing the topology on $N^{*}$. By Lemma 2 and Lemma 3, $A(D, N) \cup\{\infty\}$ is closed and equicontinuous in $C\left(D, N^{*}\right)$. According to Theorem 1 , $(N, d)$ is tight.

Suppose that $N$ is not taut. According to [3, Lemma 1.3, p. 199], there exists an integer $m$ such that $F=A\left(D^{m}, N\right)$ is not a normal family. By Lemma 2, $F \cup\{\infty\}$ is not a compact subset of $C\left(D^{m}, N^{*}\right)$. Since $(N, d)$ is tight, $F \cup\{\infty\}$ is equicontinuous; thus (Lemma 3 ) $F \cup\{\infty\}$ is not closed in $C\left(D^{m}, N^{*}\right)$. Let $\left\{f_{k}\right\}$ be a sequence in $F$ with $f_{k} \rightarrow f \in C\left(D^{m}, N^{*}\right)-(F \cup\{\infty\})$ as $k \rightarrow \infty$. Note that $f \notin C\left(D^{m}, N\right)$ $\cup\{\infty\}$. If $f(0)=\infty$, take $a \in D^{m}$ with $f(a) \neq \infty$; if $f(0) \neq \infty$, take $a \in D^{m}$ with $f(a)=\infty$. For $z \in D$, define $g_{k}(z)=f_{k}(z a /\|a\|)$ and $g(z)=f(z a /\|a\|)$. Then $g_{k} \in A(D, N), g \in C\left(D, N^{*}\right), g \notin A(D, N) \cup\{\infty\}$, and $g_{k} \rightarrow g$ as $k \rightarrow \infty$. Thus $A(D, N) \cup\{\infty\}$ is not closed in $C\left(D, N^{*}\right)$, a contradiction.

Theorem $3 .^{1}$ Let $N$ be a taut manifold. Then there exists a metric d inducing the topology on $N$ such that $(N, d)$ is tight.

1 This theorem has been obtained independently by Donald Eisenman (to appear in the Transactions of the American Society) and Peter Kiernan (to appear in the Bulletin of the American Mathematical Society) using different methods. 
Proof. Repeat the first paragraph of the proof of Theorem 2. As a sample application, we note that Theorem 3 and $[3$, Theorem D, pp. 208-209] immediately yield [3, Theorem D', pp. 208-210].

\section{REFERENCES}

1. J. L. Kelley, General topology, Van Nostrand, Princeton, N. J., 1955. MR 16, 1136.

2. S. Kobayashi and K. Nomizu, Foundations of differential geometry. Vol. I, Interscience, New York, 1963. MR 27 \#2945.

3. H. Wu, Normal families of holomorphic mappings, Acta Math. 119 (1967), 193-233. MR 37 \#468.

University of California, Riverside 